\title{
Relative growth of black holes and the stellar components of galaxies
}

\author{
N. Menci, F. Fiore, A. Bongiorno, and A. Lamastra
} INAF-Osservatorio Astronomico di Roma, via di Frascati 33, 00040 Monte Porzio Catone, Italy
e-mail: menci@oa-roma.inaf.it

Received 1 March 2016 / Accepted 30 May 2016

\begin{abstract}
Recent observations indicate that the mass of supermassive black holes (SMBHs) correlate differently with different galaxy stellar components. Comparing such observations with the results of "ab initio" galaxy formation models can provide insight on the mechanisms leading to the growth of SMBHs. Here we use a state-of-the-art semi-analytic model of galaxy formation to investigate the correlation of the different galaxy stellar components with the mass of the central SMBH. The stellar mass in the disc, in the bulge, and in the pseudo-bulge of galaxies is related to quiescent star formation, to galaxy interactions, and to the loss of angular momentum following disc instabilities, respectively. Consistently with recent findings, we find that while the predicted bulge masses are tightly correlated with the SMBH masses, the correlation between the latter and the galactic discs shows a much larger scatter, in particular when bulgeless galaxies are considered. In addition, we obtain that the predicted masses of pseudo-bulges shows little or no-correlation with the masses of SMBHs. We track the histories of merging, star formation, and SMBH accretion to investigate the physical processes at the origin of such findings within the context of cosmological models of galaxy formation. Finally, we discuss the effects of variations of our assumed fiducial model on the results.
\end{abstract}

Key words. galaxies: formation - galaxies: nuclei - galaxies: evolution

\section{Introduction}

The discovery of relations between the mass $M_{\mathrm{BH}}$ of supermassive black holes (SMBHs) and the properties of the spheroidal component of the host galaxies, like the stellar mass $M_{\text {sph }}$ (Kormendy \& Richstone 1995; Magorrian et al. 1998; Marconi \& Hunt 2003; Haring \& Rix 2004) and the stellar velocity dispersion (e.g., Ferrarese \& Merritt 2000; Gebhardt et al. 2000; Merritt \& Ferrarese 2001; Tremaine et al. 2002; Greene \& Ho 2006; Graham 2007; Gültekin et al. 2009), has shown that the growth of BHs must be tightly connected to the processes governing the evolution of galaxies. Stimulated by the above observations, a number of theoretical studies (see e.g. Kauffmann \& Haehnelt 2000; Monaco et al. 2000; Granato et al. 2004; Hopkins et al. 2006; Fontanot et al. 2006; Fanidakis et al. 2012; Hirschmann et al. 2012, 2014; Lamastra et al. 2010; Menci et al. 2003, 2014) have included the growth of SMBHs in the framework of cosmological galaxy formation models. Most of them assumed mergers and/or disc instabilities as triggers for $\mathrm{BH}$ accretion, and related the effectiveness of each accretion event to the galaxy properties (like the fraction of cold available gas, the merging rate, or the mass of the central accreting $\mathrm{BH}$ ) computed through ab-initio semi-analytic models (SAMs) of galaxy formation (see Somerville \& Davé 2015, for a review). These approaches showed that $M_{\mathrm{BH}}-M_{\mathrm{sph}}$ relations matching the observations can result in the framework of hierarchical galaxy formation models. Inclusion of sub-grid prescriptions for SMBH accretion into hydrodynamical simulations also allowed $M_{\mathrm{BH}}-M_{\text {sph }}$ relations matching the observations to be obtained, either due to self-regulation following active galactic nucleus
(AGN) feedback (in the case of Bondi accretion strongly dependent on $M_{\mathrm{BH}}$, see e.g., Di Matteo et al. 2005; Dubois et al. 2012) or to the evolution of galaxy gas content and merging rates controlling BH feeding (see, e.g., Anglés-Alcázar et al. 2013, in the case of torque-limited growth of SMBHs).

In recent years, refined observational studies of the relation between the SMBH mass and the stellar content of galaxies (e.g., Bennert et al. 2011; Graham et al. 2011; Kormendy \& Bender 2011; Kormendy et al. 2011; McConnell et al. 2011; Sani et al. 2011; Beifiori et al. 2012; Graham \& Scott 2013; McConnell \& Ma 2013) allowed the role of different physical processes to be investigated, such as ldry mergers (see, e.g., Graham \& Scott 2013), bars (see Hu 2008; Graham \& Li 2009), or disc instabilities (see, e.g., Cisternas et al. 2011), in shaping the co-evolution of BHs and host galaxies, with the aim of pinning down the basic mechanisms that trigger the growth of BHs in relation with the build-up of the different stellar populations of the host galaxies. For example, pseudo-bulges (spheroids with disc-like exponential profiles or rotational kinematics) have been reported to lie below the main relation defined by regular bulges and ellipticals (Hu 2008; Greene et al. 2010; Shankar et al. 2012), or not to correlate at all with the BH masses (Kormendy et al. 2011). Such a behaviour would have deep implications, since in the common view classical bulges are formed through violent processes such as mergers (Toomre \& Toomre 1972; Toomre 1977; Schweizer 1990; Kormendy 2013, and references therein; Avile-Reese et al. 2014) while pseudo-bulges are believed to be formed through internal evolution in isolated galaxies, involving disc instabilities, angular momentum transfer, and the ensuing inflows (see Wyse et al. 1997; Kormendy \& Kennicutt 2004; Athanassoula 2005; 
see also Gadotti 2009), though the formation of classical bulges through disc instabilities has been considered in some models (see, e.g., Perry et al. 2009; De Lucia et al. 2011) as we discuss in the final section. In addition, recent studies have shown that massive BHs can be hosted in bulgeless galaxies as shown by the presence of AGN in bulgeless galaxies (see Ho 2008; Kormendy \& Ho 2013, for reviews).

Overall, the above results suggest a scenario in which the different stellar populations (those residing in discs, in pseudobulges and in bulges) show a different degree of correlation with the BH mass (see Kormendy \& Ho 2013, for a review; for recent results based on megamaser measurements see Läsker et al. 2016). It is then interesting to investigate how such an emerging picture fits into galaxy formation models. This is particularly relevant in view of the different mechanisms that - in current models - drive the formation of the different stellar component of galaxies (see Somerville \& Davé 2015; Silk \& Mamon 2012, for recent reviews): quiescent, secular conversion of cold disc gas into stars for the disc component; major merging for classical bulges; and disc instabilities for the pseudo-bulge component. Thus, comparing the observed $\mathrm{BH}$ - host galaxy correlations for the different stellar components with results of galaxy formation models may help in understanding which of the processes involved in galaxy evolution are directly related to the growth of SMBHs.

Here we use a state-of-the-art SAM of galaxy formation to investigate the correlation of the different galaxy stellar components with the mass of the central SMBH. The model includes the physics of the galactic gas, the quiescent mode of star formation converting the cold disc gas into stars on time scales $\sim$ Gyr, the impulsive mode of star formation corresponding to starbursts triggered by interactions, and the star formation related to inflows following disc instabilities (see Hopkins 2011) implemented as described in previous papers (Menci et al. 2014; Gatti et al. 2015). The AGN feedback is computed following the expansion of the shock front through the interstellar medium as described in detail in Menci et al. (2008). In our fiducial model, the $\mathrm{BH}$ growth is triggered mainly by galaxy interactions (including fly-bys), but we also discuss a scenario where $\mathrm{BH}$ growth is entirely driven by inflows due to disc instabilities. In our model, although the AGN feedback plays a role in controlling the gas cooling and the star formation in massive galaxies, the $\mathrm{BH}$-stellar mass relation is mainly controlled by how gas feeding is related to the galaxy properties and evolution. These, in turn, relate differently with the different stellar components. The comparison with the observed relations will allow us to study the connection of the different processes driving the formation of the different stellar components (secular gas conversion, interactions, disc instabilities) with the growth of SMBHs.

\section{Semi-analytic model}

We use the semi-analytic model described in Menci et al. (2014) and Gatti et al. (2015), to which we refer for details; here we recall its key points. The merging trees of dark matter halos are generated through a Monte Carlo procedure adopting the merging rates given by the extended Press \& Schechter formalism (see Bardeen et al. 1991; Lacey \& Cole 1993; Bower 1991) starting from redshift $z=15$. We assume a cold dark matter (CDM) power spectrum of perturbations, and we adopted a total matter density parameter $\Omega_{0}=0.3$, a baryon density parameter $\Omega_{\mathrm{b}}=0.04$, a dark energy density parameter $\Omega_{\Lambda}=0.7$, and a Hubble constant $h=0.7$ in units of $100 \mathrm{~km} \mathrm{~s}^{-1} \mathrm{Mpc}^{-1}$. For each dark matter halo included in a larger halo we computed the dynamical friction process, to determine whether it will survive as a satellite, or sink to the centre to increase the mass of the central dominant galaxy; binary interactions (fly-bys and merging) among satellite sub-halos are also described by the model. The gas in each halo is initially set to have a density given by the universal baryon fraction and to be at the virial temperature. We then compute its cooling due to atomic processes. The cooled gas fraction settles into a rotationally supported disc with mass $M_{\mathrm{c}}$, disc radius $R_{\mathrm{d}}$ and disc circular velocity $V_{\mathrm{d}}$ computed as in Mo et al. (1998). The gas is converted into stars through three different channels described in Sect. 2.2: quiescent star formation, gradually converting the gas into stars over long time scales $\sim 1$ Gyr; starbursts following galaxy interactions, occurring on time scales $\lesssim 100 \mathrm{Myr}$, associated with $\mathrm{BH}$ feeding; internal disc instabilities triggering loss of angular momentum resulting in gas inflows toward the centre, therefore feeding star formation and $\mathrm{BH}$ accretion.

The energy released by the supernovae associated with the total star formation returns a fraction of the disc gas into the hot phase, providing the feedback needed to prevent overcooling. An additional source of feedback is provided by the energy radiated by the AGN which corresponds to the active accretion phase of the central BH described below in Sect. 2.1; the detailed description of our implementation of the AGN feedback is given in Menci et al. (2008). Finally, the luminosity - in different bands produced by the stellar populations of the galaxies are computed by convolving the star formation histories of the galaxy progenitors with a synthetic spectral energy distribution, which we take from Bruzual \& Charlot (2003) assuming a Salpeter IMF. The model includes the tidal stripping of the part of the stellar content of each satellite galaxy for which we adopt exactly the treatment introduced by Henriques \& Thomas (2010). We note that this is based on the computation of the tidal radius for each satellite galaxy; it is identified as the distance from the satellite centre at which the radial forces acting on it cancel out (King 1962; Binney \& Tremaine 1987; see also Taylor \& Babul 2001). These forces are the gravitational binding force of the satellite, the tidal force from the central halo and the centrifugal force. In the simple approximation of nearly circular orbits and of an isothermal halo density profile, such a radius can be expressed as $r_{\mathrm{t}} \approx \sigma_{\text {sat }} r_{\text {sat }} / \sqrt{2} \sigma_{\text {halo }}$, where $\sigma_{\text {sat }}$ and $\sigma_{\text {halo }}$ are the velocity dispersions of the satellite and of the halo, respectively, and $r_{\mathrm{sat}}$ is the halocentric radius of the satellite, that we computed in our Monte Carlo code during its orbital decay to the centre due to dynamical friction. For each satellite galaxy, the material outside this radius is assumed to be disrupted and becomes a diffuse stellar component in the host halo. Stellar stripping could play a role in producing outliers in the $M_{\mathrm{BH}}-M_{*}$ relation, as shown in the EAGLE hydrodynamical simulations by Barber et al. (2016).

\subsection{Black hole accretion}

The semi-analytic model includes $\mathrm{BH}$ growth from primordial seeds. These are assumed to originate from PopIII stars with a mass $M_{\text {seed }}=100 M_{\odot}($ Madau \& Rees 2001), and to be initially present in all galaxy progenitors. Our results are insensitive to the specific value of the seed mass as long as $M_{\text {seed }} \lesssim 10^{5} M_{\odot}$. We consider two $\mathrm{BH}$ feeding modes: accretion triggered by galaxy interactions and internal disc instabilities. These are described in detail in Menci et al. (2014) and Gatti et al. (2015), and briefly summarized below.

a) Black hole accretion triggered by interactions. The interaction rate $\tau_{\mathrm{r}}^{-1}=n_{\mathrm{T}} \Sigma\left(r_{\mathrm{t}}, v_{\mathrm{c}}, V\right) V_{\text {rel }}(V)$ for galaxies with relative 
velocity $V_{\text {rel }}$ and number density $n_{\mathrm{T}}$ in a common DM halo determines the probability for encounters, either fly-by or merging, through the corresponding cross sections $\Sigma$ given in Menci et al. (2002, 2014). The fraction of gas destabilized in each interaction corresponds to the loss $\Delta j$ of orbital angular momentum $j$, and depends on the mass ratio of the merging partners $M^{\prime} / M$ and on the impact factor $b$ as follows:

$f \approx \frac{1}{2}\left|\frac{\Delta j}{j}\right|=\frac{1}{2}\left\langle\frac{M^{\prime}}{M} \frac{R_{\mathrm{d}}}{b} \frac{V_{\mathrm{d}}}{V_{\mathrm{rel}}}\right\rangle$,

We assume that in each interaction $1 / 4$ of the destabilized gas mass $f M_{\mathrm{c}}$ feeds the central $\mathrm{BH}$, while the remaining fraction feeds the circumnuclear starbursts (Sanders \& Mirabel 1996). Thus, the $\mathrm{BH}$ accretion rate is given by

$$
\frac{\mathrm{d} M_{\mathrm{BH}}}{\mathrm{d} t}=\frac{1}{4} \frac{f M_{\mathrm{c}}}{\tau_{\mathrm{d}}}
$$

where the time scale $\tau_{\mathrm{d}}=R_{\mathrm{d}} / V_{\mathrm{d}}$ is assumed to be the crossing time of the galactic disc.

b) Black hole accretion induced by disc instabilities. As in other SAMs (see, e.g., Lo Faro et al. 2009; De Lucia et al. 2011; Hirschmann et al. 2012), we assume that they to arise in galaxies with disc mass exceeding $M_{\text {crit }}=v_{\max }^{2} R_{\mathrm{d}} / G \epsilon$ (Efstathiou et al. 1982) with $\epsilon=0.75$, where $v_{\max }$ is the maximum circular velocity associated with each halo (Mo et al. 1998). This criterion strongly lowers the probability that disc instabilities occur in massive, gas-poor galaxies, and also in dwarf galaxies characterized by small values of the gas-to-DM mass ratios. The instabilities induce loss of angular momentum resulting in strong inflows that we compute following the description in Hopkins et al. (2011), recast and extended as in Menci et al. (2014). The resulting $\mathrm{BH}$ accretion rate (in units of $M_{\odot} \mathrm{yr}^{-1}$ ) is

$$
\begin{aligned}
& \frac{\mathrm{d} M_{\mathrm{BH}}}{\mathrm{d} t} \approx \frac{\alpha f_{\mathrm{d}}^{4 / 3}}{1+2.5 f_{\mathrm{d}}^{-4 / 3}\left(1+f_{0} / f_{\mathrm{gas}}\right)} \times\left(\frac{M_{\mathrm{BH}}}{10^{8} M_{\odot}}\right)^{1 / 6}\left(\frac{M_{\mathrm{d}}}{10^{9} M_{\odot}}\right) \\
& \text { with } f_{0} \approx 0.2 f_{\mathrm{d}}^{2}\left[\frac{M_{\mathrm{d}}}{10^{9} M_{\odot}}\right]^{-1 / 3} \quad f_{\mathrm{gas}} \equiv \frac{M_{\mathrm{gas}}}{M_{\mathrm{d}}} .
\end{aligned}
$$

Here $M_{\mathrm{BH}}$ is the central black hole (BH) mass, $f_{\mathrm{d}}$ is the total disc mass fraction, and $M_{\mathrm{d}}$ and $M_{\mathrm{gas}}$ the disc and the gas mass calculated at the reference radius $R_{0}=100 \mathrm{pc}$ (writing the above equation in terms of different reference radius $R_{0}$ would not change the resulting accretion rate, see Menci et al. 2014). The constant $\alpha$ depends on the exact form of the assumed disc surface density profile and to the shape of the Schmidt star formation law in the inner region (see Hopkins et al. 2011). Present observational uncertainties in such quantities yield an allowed range $1 \leq \alpha \leq 5$. In the following we take $\alpha=5$; this value approximates that corresponding to our assumed Kennicutt-Schmidt law relation $\dot{M}_{*} \propto M_{\mathrm{c}} / \tau_{\mathrm{d}}$ with a precision better than $5 \%$ (see Eq. (A.14) in Menci et al. 2014). We note that the expression in Eq. (3) assumes the persistence of a stable disc (it is derived in the WKB approximation; see Hopkins \& Quataert 2011; and Menci et al. 2014) and hence does not hold in our major merging events. In addition, the accretion rate in Eq. (3) has a mild dependence on the $\mathrm{BH}$ mass, but depends very strongly on the disc mass fraction and on the gas mass fractions ( $f_{\mathrm{d}}$ and $\left.f_{\text {gas }}\right)$. Thus, we expect disc instabilities to be effective in gas-rich systems which underwent a limited number of merging events during their past history.
In our model, at any given time BHs are allowed to grow via both channels. As discussed in Menci et al. (2014) the build-up of the final $\mathrm{BH}$ masses is dominated by the interaction-driven accretion (see Sect. 2.3 below and Fig. 2). However, inflows produced by disc instabilities contribute to the star formation of the galaxy and drive the growth of the pseudo-bulge, as we discuss below.

\subsection{Star formation}

Our model includes three modes of star formation:

i) The quiescent mode, corresponding to the gradual conversion of disc gas into stars with a rate $\dot{M}_{*} \propto M_{\mathrm{c}} / \tau_{\mathrm{d}}$ directly related to the disc time defined above. The proportionality constant is given by the measured local Schmidt-Kennicutt law yielding a conversion time $\tau_{*}(z=0)=1$ Gyr (e.g., Santini et al. 2014). The stars formed through this channel are assigned to the galactic disc.

ii) The impulsive mode, corresponding to starbursts triggered by galaxy interactions: the interaction rate is that given at point a) in Sect. 2.1. The fraction of gas converted into stars corresponds to $3 / 4 f$ where the destabilized gas fraction $f$ is that given in Eq. (2.1). Following Hopkins et al. (2009) we assume that in mergers with mass ratio $\mu \geq 0.2$ a fraction $1-f_{\text {gas }}$ of the disc mass is transferred to the bulge, while the stars formed in minor mergers and fly-bys are assigned to the disc. The resulting distribution of bulge-to-total $(B / T)$ stellar mass ratio has been tested against observations in Menci et al. (2014). We note, however, that our results do not change appreciably if we take the canonical assumption that all disc stars are transferred into the bulge during major mergers.

iii) Disc instabilities. These arise when the disc mass exceeding $M_{\text {crit }}=v_{\max }^{2} R_{\mathrm{d}} / G \epsilon$, as discussed in Sect. 2.1b. The corresponding loss of angular momentum induces nuclear star formation. Since the model in Hopkins et al. (2011) assumes an equilibrium between the mass inflow and star formation, the latter reads $\dot{M}_{*}=A_{*} \dot{M}_{\mathrm{BH}, \mathrm{DI}}$ (see Menci et al. 2014, for the computation), where $\dot{M}_{\mathrm{BH}, \mathrm{DI}}$ is the $\mathrm{BH}$ accretion rate in Eq. (3). The exact value of the proportionality constant $A_{*} \geq 10^{2}$ depends on the detailed radial profile of the disc potential and of the gas surface density. In Menci et al. (2014) we obtain a value $A_{*} \approx 100$ in the nuclear region of the galaxy, while the contribution from the outer disc regions may yield an additional $\sim 50 \%$ the nuclear value in the case of an exponential density profile. In the following we keep the value $A_{*} \approx 100$ as our fiducial choice; larger values would result in a slight shift of the pseudo-bulge masses, and would not change our main results (see below and Sect. 3). The stars formed through the disc instability mode are assigned to a pseudo-bulge component, according to a widely adopted view (see, e.g., Combes et al. 1990; Raha et al. 1991; Debattista et al. 2006; Kormendy \& Kennicutt 2004; Athanassoula et al. 2005; see also Mendez-Abreu 2014; Obreja et al. 2013) also implemented in other SAMs (see e.g. Fontanot et al. 2015a). However, we note that alternative explanations (that we do not consider here) have been proposed; e.g., Eliche-Moral et al. (2011) suggested that pseudo-bulges might also be created by the secular accretion of low-density satellites into the main galaxy, while Keselman \& Nusser (2012) propose a merger origin for pseudo-bulges. In our implementation, a star formation mode of this nature requires large fraction of gas left available in the galaxy, so that the gas mass can exceed the mass threshold $M_{\text {crit }}$ (Sect. 2.1) for the onset of disc instabilities, and to allow 

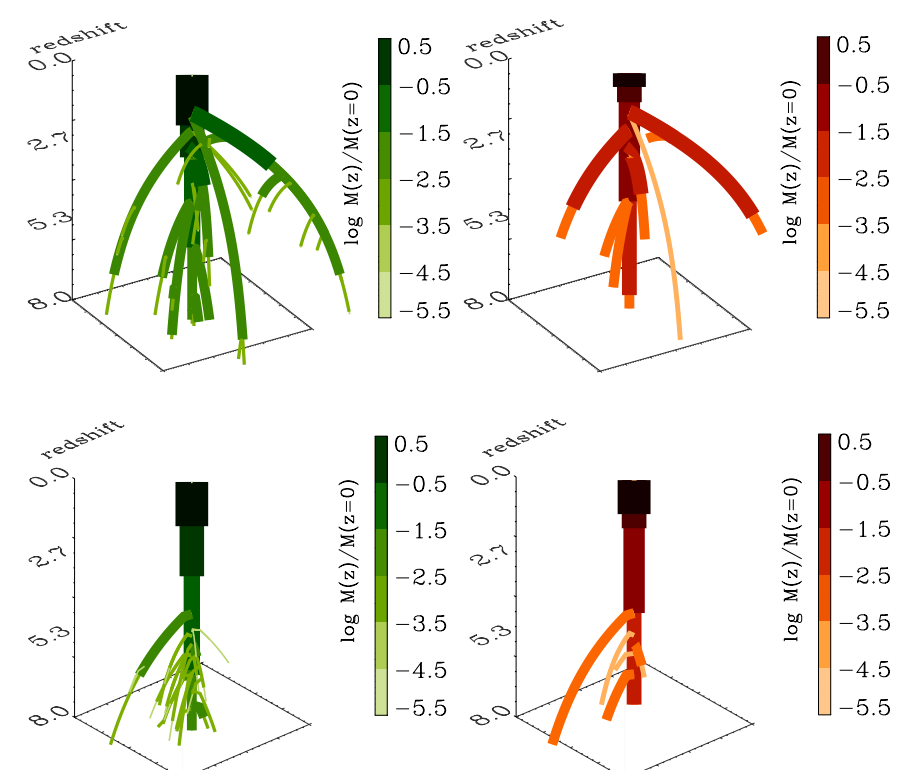

Fig. 1. Selected merging trees. Each branch represents a progenitor of the final galaxy at the redshift indicated by the vertical axis. The spatial distribution of branches in the $x-y$ plane is arbitrary, chosen to separate branches in order to obtain a better graphical visualization. The colour and size of each branch correspond to the stellar mass (left panels) and $\mathrm{BH}$ mass (right panels) associated with the progenitor galaxies. The corresponding logarithmic mass scale in units of the final mass is shown in the colourbars. To avoid overcrowding in the figure, we show only branches with masses larger than $10^{-5}$ the final value at $z=0$. Thus, accretion of the small lumps containing the seed $\mathrm{BH}$ masses is not represented. The first history leads to a final galaxy with $B / T=0.5$, the second to a final elliptical galaxy with $B / T=0.9$.

for large values of the disc and gas fractions $f_{\mathrm{d}}$ and $f_{\text {gas }}$ entering the accretion rate in Eq. (3).

Each model galaxy is allowed to have both a bulge and a pseudobulge. When compared with data concerning pseudo-bulges we consider model galaxies where such stellar component dominates over the classical bulge component.

\subsection{Relative growth of black hole and total stellar content of galaxies}

The model allows the $\mathrm{BH}$ accretion (Sect. 2.1) and star formation (Sect. 2.2) to be followed in all the progenitors along the merger history of any given final galaxy. In Fig. 1 we show how the growth histories of $M_{*}$ and $M_{\mathrm{BH}}$ are distributed among the progenitors of two selected final $(z=0)$ galaxies in our SAM (with mass $M_{*} \approx 10^{11} M_{\odot}$ ). The merger trees result from our Monte Carlo realizations described above (see the beginning of Sect. 2), and include all the dynamical processes affecting the evolution of sub-halos. Each branch represents a progenitor of the final galaxy at the redshift indicated by the vertical axis, and the size and colour correspond to the fractional mass (normalized to the final mass) of the stellar (left panel) and BH (right panel) component of each progenitor resulting from the full computation of our SAM.

We note that, while at high redshift the stellar mass is distributed among numerous progenitors (since there is also a contribution from the quiescent mode that does not depend on galaxy merging or interactions), the contributions to the final $\mathrm{BH}$ mass are mainly in the main progenitor and in the few main
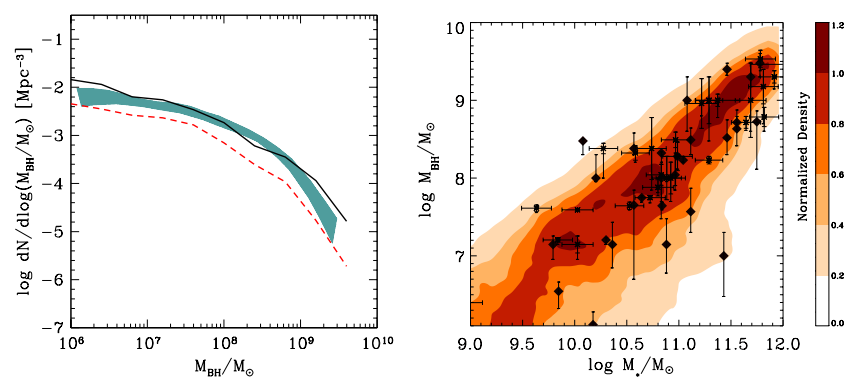

Fig. 2. a) (Left) black hole mass function at $z<0.5$ built up by interaction-driven accretion (solid line) and by $\mathrm{BH}$ accretion due to disc instabilities (dashed line). The shaded region defines the spread in observational estimates obtained using different methods, as compiled by Shankar et al. (2009). b) (Right) local relation between the BH mass and the total stellar content of galaxies is compared with data. Data points represent the observed local relation from Häring \& Rix (2004, diamonds), and Marconi \& Hunt (2003, squares; here $M_{*}$ is derived using the best-fitting virial relation of Cappellari et al. 2006); the colour code represents the fraction of objects as a function of $M_{\mathrm{BH}}$ for any given value of $M_{*}$, as indicated by the bar.

branches where most of the merging activity is concentrated. The merging activity takes place mainly at high redshifts $\gtrsim 3$.

The statistical effect of all the growth histories results in the final $\mathrm{BH}$ mass distribution shown in Fig. 2a and in the local relation between the $\mathrm{BH}$ mass and the total stellar mass in Fig. $2 \mathrm{~b}$.

In Fig. 2a, the contributions from interaction-driven accretion and disc instabilities are also shown, to show how the former constitutes the dominant mode of BH growth, while Fig. $2 b$ illustrates the scatter that characterizes the relation when the total stellar mass of the host galaxy is considered as being due to the combined effect of the growth histories shown in Fig. 1. However, selecting final galaxies with a specific dominant stellar component corresponds to selecting growth histories with specific characteristics, yielding different relations between the $\mathrm{BH}$ mass and the selected stellar component. In the next section, we dissect the stellar mass of host galaxies in the different components discussed above, to pin down the main source of the scatter in Fig. $1 b$ in relation to the growth histories, and to investigate the role played by the different physical processes described in this section to the build-up of the BH mass.

\section{Results}

Here we present the predicted correlation between the $\mathrm{BH}$ mass and the different galaxy components. We compare our results with the observations presented in Kormendy \& Ho (2013), who collected available measurements of such quantities for a sample of 45 elliptical galaxies and 43 spiral and S0 galaxies; the latter include 21 galaxies with classical bulge and 22 with pseudobulges. The BH mass measurements are based on different methods: stellar dynamics, CO molecular gas disc dynamics, maser disc dynamics, ionized gas dynamics. The last of these, however, has been considered only when the observations took into account broad emission line widths (see the above authors for details), and this contributes to the larger normalization with respect to early works (see, e.g., Marconi \& Hunt 2003). Although the sample represents a substantial collection of updated measurements, it is important to note that it does not constitute a homogeneous sample with well-defined selection criteria, and in the following we do not attempt to perform statistical analysis concerning the relative abundance of $\mathrm{BH}$ found in the different galactic components (for the AGN luminosity functions, 
BH mass function, and the $B / T$ distributions predicted by our model see Menci et al. 2014). We instead compare the data with the relation between $M_{\mathrm{BH}}$ and the stellar mass of different components resulting in our model.

The observed $M_{\mathrm{BH}}-M_{*, B}$ correlation between the BH mass and the mass of the classical bulge component is shown in Fig. 3 and compared with the prediction of our model for the same component (built up by major mergers, Sect. 2.2, point ii), for galaxies in the same magnitude range $-24 \leq M_{K} \leq-21$ spanned by the observations. Within a standard deviation, the distribution we obtain is consistent with a single linear dependence in the $\log M_{\mathrm{BH}}-\log M_{*, B}$ plane. The slope, the normalization, and the scatter of the model predictions are consistent with the observations. Indeed, a linear regression analysis of the predicted $\log M_{\mathrm{BH}}-\log M_{*, B}$ distribution yields $\log M_{\mathrm{BH}} / M_{\odot}=$ $(1.15 \pm 0.1) \log \left(M_{* . B} / M_{\odot}\right)-3.84 \pm 1$, remarkably close to that derived by Kormendy \& Ho (2013) for the data sample $\log M_{\mathrm{BH}} / M_{\odot}=(1.16 \pm 0.08) \log \left(M_{* . B} / M_{\odot}\right)-4.1 \pm 1$, but flatter than the intrinsic relation recently proposed by Shankar et al. (2016) on the basis of their analysis of the selection bias; this is a non-linear relation, with a logarithmic slope $\approx 1.9$ and a normalization close to that in Kormendy \& Ho (2013) at the large-mass end $\left(M_{*, B} \approx 10^{11.5} M_{\odot}\right)$ of the $M_{\mathrm{BH}}-M_{*, B}$ relation. The scatter we obtain ( 0.4 dex, mildly dependent on the bulge mass, see the top histogram in Fig. 3) is slightly larger than the intrinsic scatter of the data sample $\approx 0.3$ dex (see Kormendy \& Ho 2013). We note that the exact value of the normalization in the predicted $\log M_{\mathrm{BH}}-\log M_{*, B}$ relation, although similar to the value measured by Kormendy \& Ho (2013), is not a true prediction of our model; in fact, it depends on the assumed ratio of $\mathrm{BH}$ accretion to nuclear star formation, a free quantity for which we adopted the fiducial value 1/4 (Sect. 2.1 and Eq. (1)).

The almost linear behaviour constitutes a natural outcome in a scenario where the interaction-driven accretion stimulates both nuclear star formation and $\mathrm{BH}$ accretion, as already shown in early SAMs with tunable accretion efficiency (Kauffmann \& Haenhelt 2000; Cattaneo et al. 2005; Croton et al. 2006). However, approximately linear $\log M_{\mathrm{BH}}-\log M_{*, B}$ relation also arise in several hydrodynamical simulations (see, e.g., Di Matteo et al. 2005; for recent cosmological simulations see Sijacki et al. 2015; Volonteri et al. 2016) which assume BHs to grow through Bondi accretion. In such simulations the fast runaway in the $\mathrm{BH}$ mass produced by the quadratic dependence on $M_{\mathrm{BH}}$ of the accretion rate is counteracted by strong feedback to produce $M_{\mathrm{BH}}-M_{*, B}$ relations close to the observed one, with a logarithmic slope that ranges from 0.75 to 1.25 , also depending on the adopted method to measure the bulge mass in the simulations. Thus, the slope in the $M_{\mathrm{BH}}-M_{*, B}$ relation does not constitute a sensible probe to pin down the physical origin of the observed $M_{\mathrm{BH}}-M_{*, B}$ relation. In this context, studying how the growth of BHs and of the different stellar components are connected may help to provide constraints on the physical origin of the BH-galaxy co-evolution.

To this aim, we start studying the paths followed by the galaxy progenitors in the build-up of the local relations. For the case of the $M_{\mathrm{BH}}-M_{*, B}$ relation, these are presented in Fig. 3 (bottom panel). This shows the time evolution of $M_{*, B}$ and $M_{\mathrm{BH}}$ contributed by all progenitors of a few selected final galaxies, from $z=8.3$ to the final position in the $\log M_{\mathrm{BH}}-\log M_{*, B}$ relation shown in the contour plot on the top. The paths have been chosen so they end up in different regions of the final $M_{\mathrm{BH}^{-}} M_{*, B}$ relation corresponding to final galaxies with $10^{9} M_{\odot} \leq M_{*, B} \leq 10^{11} M_{\odot}$ (we note that the final part of the trajectories is covered by the top-most plane). In the figure, the circles mark the onset of a

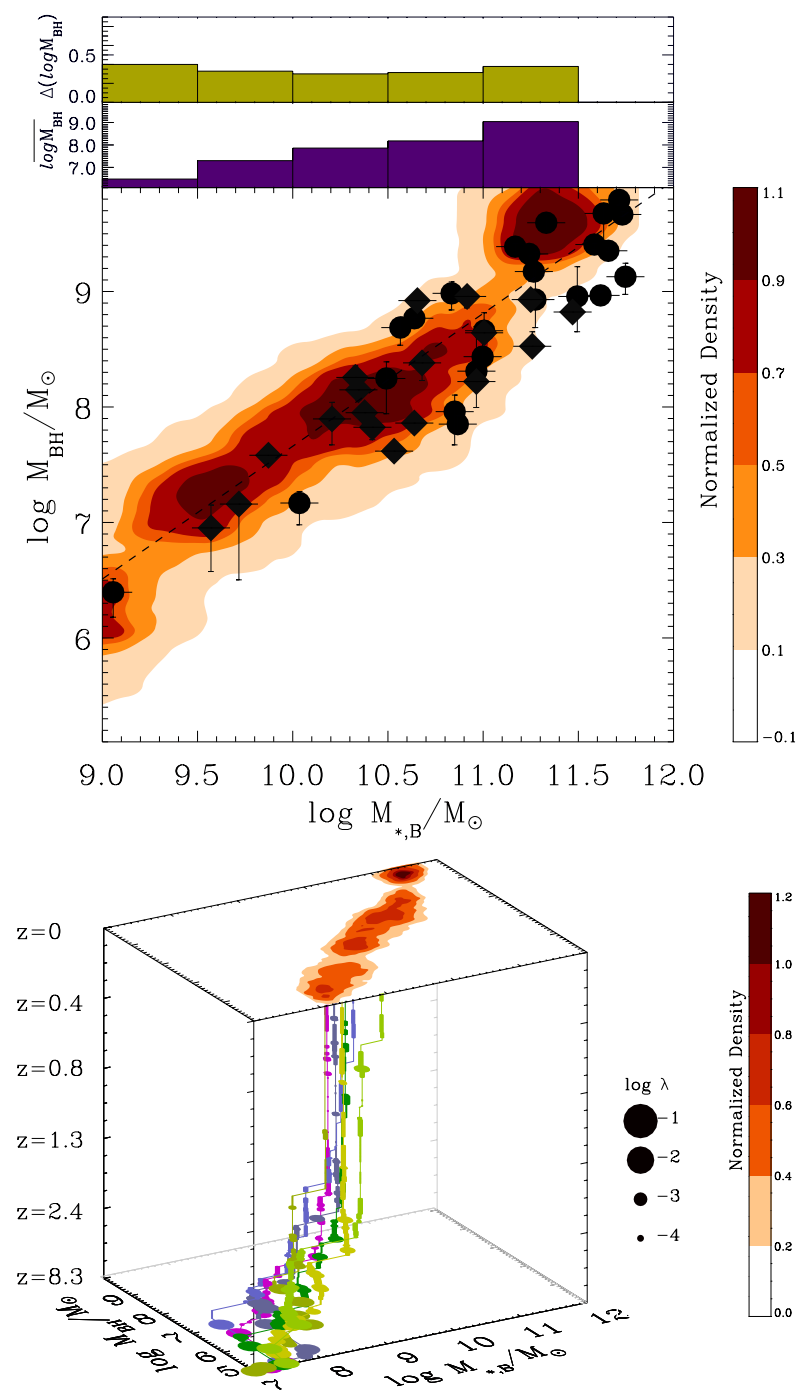

Fig. 3. Upper panel: contour plot showing the predicted local relation $M_{\mathrm{BH}}-M_{*, B}$ between the BH mass and the classical bulge mass. The contours indicate the number of galaxies in a $M_{\mathrm{BH}}-M_{*, B}$ bin, normalized to the total number at fixed $M_{*, B}$ as shown in the colour bar. We compare these values with the data reported in Kormendy \& Ho (2013) for ellipticals (circles) and classical bulges (diamonds) in spiral galaxies. The dashed line in the contour plot shows the fit $\log M_{\mathrm{BH}} / M_{\odot}=1.15 \log \left(M_{* . B} / M_{\odot}\right)-3.84$, while the histograms on the top show the average value $\overline{\log M_{\mathrm{BH}}}$ and the scatter $\Delta \log M_{\mathrm{BH}}$ of the distribution of $\log M_{\mathrm{BH}} / M_{\odot}$ in different bins of bulge stellar mass. Lower panel: each coloured path shows the time evolution of the $\mathrm{BH}$ mass $M_{\mathrm{BH}}(t)$ and bulge mass $M_{* B}(t)$ contributed by all progenitors of a few selected final galaxies. The circles show the onset of an active AGN phase; their size corresponds to the Eddington ratio $\lambda \equiv \dot{M}_{\mathrm{BH}} / \dot{M}_{\mathrm{Edd}}$ as shown in the legend.

$\mathrm{BH}$ accretion phase (AGN), and their sizes correspond to the Eddington ratio (see caption and legend).

Interactions are the leading trigger for $\mathrm{BH}$ accretion in our model (see Sects. 2.1-2.3 and Fig. 2), so they drive the growth of both the BH and bulge mass. Since in CDM models interactions between comparable clumps (those most effective in triggering starbursts and $\mathrm{BH}$ growth, see Eq. (1)) take place mainly at high redshifts (see, e.g., Zhao et al. 2003), the growth histories are characterized by a rapid increase in $M_{\mathrm{BH}}$ and $M_{*, B}$ in a short interval of cosmic time $(z \gtrsim 2.5)$, after which most of the gas has been turned into stars, leaving passively evolving bulges 


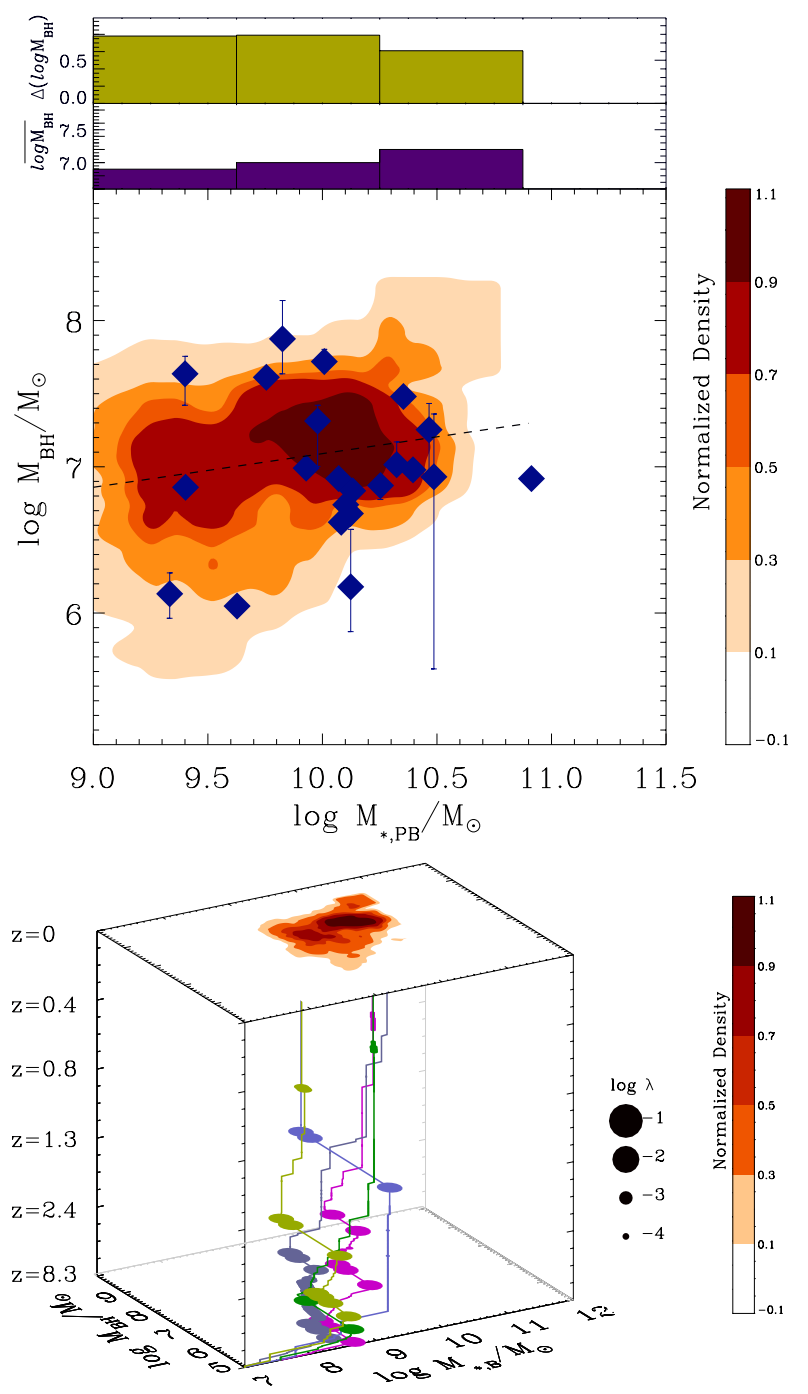

Fig. 4. As in Fig. 3, but here we consider the relation between the $\mathrm{BH}$ mass $M_{\mathrm{BH}}$ and the mass of the pseudo-bulge component $M_{*, \mathrm{~PB}}$, for galaxies where the latter dominates over the classical bulge component. The dashed line in the top panel corresponds to a linear fit $\log M_{\mathrm{BH}} / M_{\odot}=0.23 \log \left(M_{* . B} / M_{\odot}\right)+4.79$.

where only occasional events will trigger minor $\mathrm{BH}$ accretion. The short time lapse when the same physical process triggers the growth of both $M_{\mathrm{BH}}$ and $M_{*, B}$ results in a final tight correlation as we show later.

A different situation occurs when pseudo-bulges are considered. In Fig. 4 we show the distribution of $\mathrm{BH}$ masses as a function of the pseudo-bulge stellar mass $M_{*, \mathrm{~PB}}$ of the host, for the model galaxies where such a stellar component dominates over the classical bulge component, to compare with the data obtained under similar selection criteria (see Kormendy \& Ho 2013).

Compared to the previous case in Fig. 3, a much weaker correlation (with a logarithmic slope 0.23 ) and a larger scatter $\sim 0.7$ are obtained. In our model, pseudo-bulges originate from the loss of angular momentum due to disc instabilities (Sect. 2.2, point iii), so that the normalization of the computed magnitude depends on the parameter $A_{*}$ which determines the normalization of such a star formation mode (Sect. 2.2); such a parameter, in turn, depends on the density profile assumed for the outer disc region. Following our discussion in Sect. 2.2, for an exponential profile a horizontal shift up to 0.5 can affect the $M_{K}$ of the computed galaxies. However, this does not affect our main point here, namely, that the appreciably larger dispersion of the distribution compared to that obtained for classical bulges (as shown by the larger scatter, shown in the top histogram of Fig. 4) is in agreement with the observed scatter shown by the data in Kormendy \& Ho (2013); disc instabilities - which build up the pseudobulge component in our model - provide only a minor contribution to the growth of SMBHs (see Fig. 2a), so that the growth of pseudo-bulges is in practice uncorrelated to that of most of the BH mass, as shown by the paths in the bottom panel of Fig. 4. The same growth paths also show that, since disc instabilities require large gas fractions (Eq. (3)), the growth of a substantial pseudo-bulge must take place along those histories characterized by a low number of major merging at high redshifts, in order to avoid the consumption of gas occurring in major mergers at early times. Thus, the paths leading to $\mathrm{BH}$ in galaxies with a dominant pseudo-bulge are characterized by (a) BH growth largely uncorrelated to the growth of the pseudobulge; and (b) the formation of the stellar mass $\Delta M_{*}$ spread out over a much wider arc of time compared to classical bulges, with a much smaller number of merging events $N$. The combination of (a) and (b) result in a larger dispersion for $\log M_{\mathrm{BH}}$ (for a more extended interpretation, see the Discussion in the next section).

We note that switching off interactions would indeed restore an almost linear $M_{\mathrm{BH}}-M_{*, \mathrm{~PB}}$ correlation (as shown in Menci et al. 2014; see also Angles-Alcazar et al. 2016), although this would result into an evolution of the AGN luminosity functions not matching the observations at both the faint and the bright end.

An interesting consequence of the interplay between the different star formation modes and the growth of BHs resulting from the modelling in Sect. 2 is the predicted weak correlation between $\mathrm{BH}$ mass and the stellar disc component, as is shown in Fig. 5. This shows the distribution of $\mathrm{BH}$ masses as a function of the $K$-band magnitude $M_{K \text {,disc }}$ (in direct comparison with available data) of the disc stellar component of our simulated galaxies. The relation we find is characterized by a large scatter that is broadly consistent with the dispersion of the data points. Again, the origin of the model results can be traced back to the growth of the BHs in relation to the star formation history of the disc component, shown in the bottom panel. While the $\mathrm{BH}$ growth is mainly triggered by interactions which rapidly increase the $\mathrm{BH}$ mass on a very short time scale $\sim \tau_{\mathrm{d}}$, this process is not directly correlated with the growth of the stellar disc component, which is due to the quiescent gas conversion (point (i) in Sect. 2.2) which takes place over a much longer time scale $\sim$ Gyr. Such a slow gas conversion is responsible for the continuous growth of the stellar mass (at fixed BH mass) shown by the paths in the bottom panel of Fig. 5 .

We note that an effect of this nature for galaxies with a prominent disc component is deeply rooted in the cosmological properties of galaxy formation. In fact, such galaxies must have formed in lower density regions (compared to bulge-dominated systems), where the milder interaction rate allows the persistence of the disc. This results in lower star formation rates at high redshifts $z>4$, leaving a larger reservoir of cold gas available for accretion at later cosmic times, thus allowing for a prolonged accretion phase at lower redshifts $z<2$.

An extreme instance of the above behaviour is constituted by bulgeless galaxies. These have been selected from the Mock catalogue generated by our SAM as those characterized by bulgeto-total ratio $B / T \leq 0.1$. The corresponding distribution of the hosted BH masses as a function of the disc $K$-band magnitude is shown in Fig. 6, and compared with the data presented in 


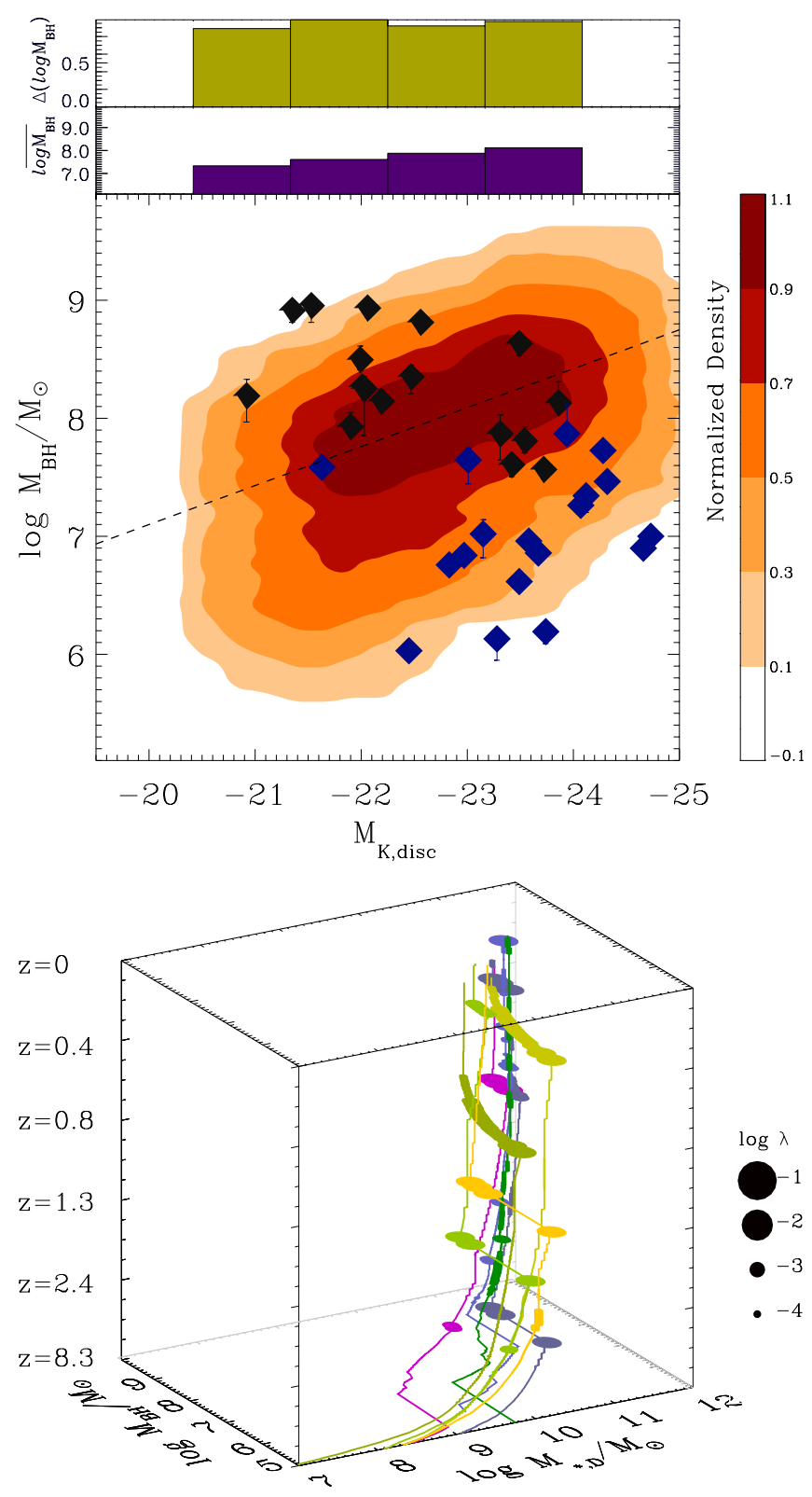

Fig. 5. Upper panel: predicted distribution of galaxies in the $M_{\mathrm{BH}}-$ $M_{K \text {,disc }}$ plane from our SAM (contour plot), where $M_{K \text {,disc }}$ is the $K$-band magnitude of the stellar disc. The data are from Kormendy \& Ho (2013): black diamonds refer to spiral galaxies with classical bulges, while blue diamonds correspond to galaxies with a pseudo-bulge. The dashed line in the contour plot shows the linear fit $\log M_{\mathrm{BH}} / M_{\odot}=$ $-0.264 \log \left(M_{K \text {,disc }} / M_{\odot}\right)+1.97$, while the histograms on the top show the average value $\overline{\log M_{\mathrm{BH}}}$ and the scatter $\Delta \log M_{\mathrm{BH}}$ of the distribution of $\log M_{\mathrm{BH}} / M_{\odot}$. Lower panel: each coloured path shows the time evolution of the BH mass $M_{\mathrm{BH}}(t)$ and disc mass $M_{*, \mathrm{D}}(t)$ contributed by all progenitors of a few selected galaxies. The circles show the onset of an active AGN phase; their size corresponds to the logarithm of the Eddington ratio $\lambda \equiv \dot{M}_{\mathrm{BH}} / \dot{M}_{\mathrm{Edd}}$ as shown in the legend.

Kormendy \& Ho (2013). We note that the requirement of low $B / T \leq 0.1$ translates into selecting model galaxies originating from those (rare) histories characterized by the absence of major merging at large redshifts. In this case, the $\mathrm{BH}$ growth is due to the later fly-by or minor merging events that do not lead to bulge formation. In this case the $\mathrm{BH}$ growth is is not only uncorrelated
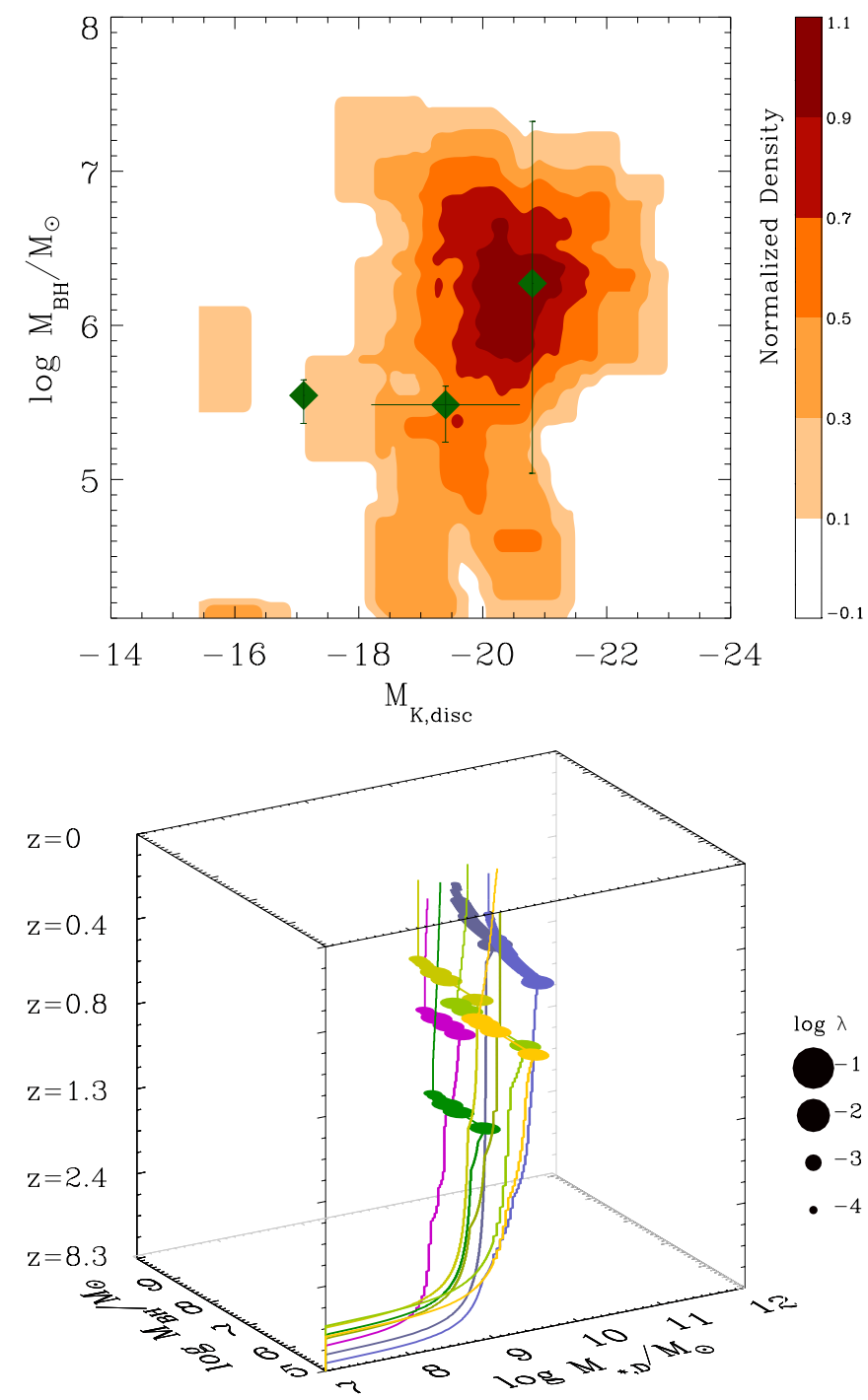

Fig. 6. As in Fig. 5, but for galaxies with no bulge. Data are taken from Kormendy \& Ho (2013), and are based on observations of NGC 4395 (Peterson et al. 2005), Pox53 (Thornton et al. 2008), and Henize 2-10 (Reines et al. 2011).

with the growth of the disc component, but is also spread over a large range of cosmic times $z \lesssim 1$ since high redshift mergers are excluded by definition. The result is a highly dispersed $M_{\mathrm{BH}}-M_{K}$ relation. Its real consistence with the observational distribution must await more homogeneous observational samples with larger statistics and - more importantly - well-defined selection criteria.

The different behaviour of the $\mathrm{BH}$ mass distribution in bulges, pseudo-bulges, and discs is compared and summarized in Fig. 7. In the figure we plot the distribution function of $\Delta \log M_{\mathrm{BH}}$, the offset of the $\mathrm{BH}$ mass from the value where the $M_{\mathrm{BH}}-M_{*}$ relation is peaked, for a reference stellar mass $M_{*}=2 \times 10^{10} M_{\odot}$. In addition to the appreciably larger dispersion obtained for the $\mathrm{BH}$ distribution in pseudo-bulges and discs than in bulges, we note that for the first two the distributions are skewed toward lower BH masses. This might explain the observational findings concerning the steepening of the global $M_{\mathrm{BH}}-M_{\text {sph }}$ relation for small values of $M_{\mathrm{BH}}$ reported by some authors (see Graham \& Scott 2015, and references therein). 


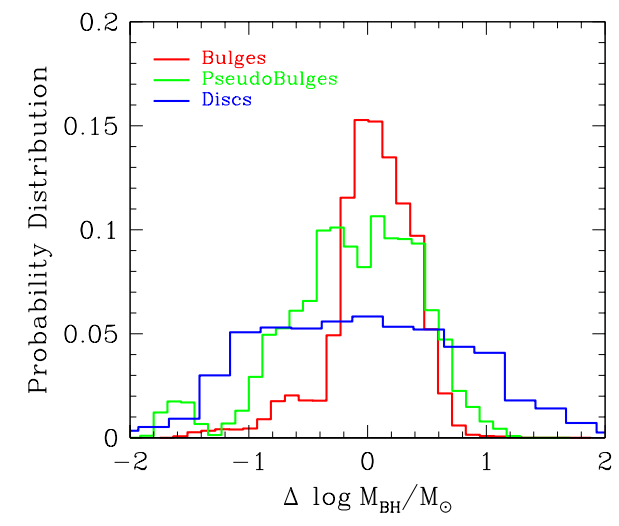

Fig. 7. Predicted distributions of $\log M_{\mathrm{BH}}$ around the peak value at a stellar mass $M_{*}=2 \times 10^{10} M_{\odot}$ is shown for bulges, pseudo-bulges and discs (red, green, and blue histogram, respectively).

\section{Discussion}

To better understand the origin of the small scatter in the relation in Fig. 3 compared to that in Figs. 4 and 5, we considered a simplified rendition of the processes considered in our SAM. Specifically, we consider a toy model (described in detail in the Appendix), where the build-up of a given stellar mass $\Delta M_{*}$ in a time $\Delta t$ is contributed by (i) the merging of galaxies containing stellar and $\mathrm{BH}$ masses; (ii) a term correlated to the $\mathrm{BH}$ growth, corresponding to impulsive star formation (starbursts) proportional (though a constant $C$ ) to the $\mathrm{BH}$ accretion rate. This term mimics the effects of merging and of the interactions, which in our model trigger $\mathrm{BH}$ accretion proportional to the star bursts, see Sect. 2; (iii) a term uncorrelated to the $\mathrm{BH}$ growth, with a star formation rate described in terms of a stochastic variable $\epsilon$. This represents the growth of the stellar mass due to secular processes not related to the $\mathrm{BH}$ growth (Sect. 2.2), with the stochastic term $\epsilon$ reflecting the different content of cold gas that can be found in galaxies due to their different merging histories and formation times (e.g., for the quiescent star formation $\epsilon \propto M_{\mathrm{c}} / \tau_{\mathrm{d}}$, see point $i$ in Sect. 2.2). The above toy model allows the analytical computation analytically (Appendix A) of how the variance $\sigma\left(\log M_{\mathrm{BH}, \mathrm{f}}\right)$ of the distribution of final logarithmic $\mathrm{BH}$ masses $\log M_{\mathrm{BH}, \mathrm{f}}$ is related to the initial value $\sigma\left(\log M_{\mathrm{BH}, \mathrm{i}}\right)$ associated with the distribution of progenitor galaxies after $N$ merging events within the interval $\Delta t$. Such a toy model extends the simple case explored by Peng (2007) which considered only the effect of merging on the variance of the $M_{\mathrm{BH}}$ distribution. In the limit of a large number of merging events $N$ we obtain (see Appendix A)

$\sigma\left(\log M_{\mathrm{BH} f}\right)=\frac{\sigma\left(\log M_{\mathrm{BH}, \mathrm{i}}\right)}{\sqrt{N}}+\frac{C \sigma(\epsilon) \Delta t}{N \overline{M_{\mathrm{BH}, \mathrm{i}}}}$

where $\overline{M_{\mathrm{BH}, \mathrm{i}}}$ denotes the average of the initial $\mathrm{BH}$ mass distribution, and $\sigma(\epsilon)$ is the variance generated by all star formation processes not correlated to the $\mathrm{BH}$ accretion. The first term in Eq. (5) is the same obtained by Peng (2007) and describes the statistical effect of merging, which yields a fractional scatter that decreases during the growth of the stellar mass as $1 / \sqrt{N}$ simply due to law of large numbers. The second term in Eq. (4) arises only in the presence of $\mathrm{BH}$ accretion $(C \neq 0)$. It vanishes for star formation entirely correlated with the BH growth $(\sigma(\epsilon) \rightarrow 0)$, and increases with the duration $\Delta t / N$ corresponding to the build-up of the stellar mass $\Delta M_{*}$.
In the case of the build-up of classical bulges through merging events shown in Fig. 3, $N$ takes large values during a small time lapse $\Delta t$, because at high redshifts hierarchical models predict fast, numerous interactions to take place in a small time lapse $\Delta t$, corresponding to a fast build-up of the stellar mass $\Delta M_{*}$. Following Eq. (4), this implies that the effect of the term corresponding to the growth of $\mathrm{BH}$ uncorrelated with the stellar mass is suppressed, and that the large number of merging events $N$ leads to a rapid decrease in the scatter in $\log M_{\mathrm{BH}}$, as in fact is shown by the paths in the bottom panel of Fig. $3 \mathrm{~b}$.

Equation (4) can be used for a more quantitative interpretation of the origin of the scatter in Figs. 3-5. While, of course, the quantities entering the toy model in Eq. (4) can be estimated only approximately (an exact computation of the $\mathrm{BH}$ accretion, star formation and merging processes requires the full semi-analytic computation), we compute a first-order estimate of the scatter expected from Eq. (4) to provide a guideline to understanding how the quantities characterizing the growth histories in the bottom panels of Figs. 3-5 (number of mergers, time scales for the growth of BHs, and stellar masses) determine the final scatter in the correlation between $M_{\mathrm{BH}}$ and the bulge, pseudo-bulge, and disc stellar masses.

To this end, we start from the case of classical bulges. We first compute $N$ corresponding to the growth paths in Fig. 3 (bottom panel). For $z \geq 3$, our SAM yields values ranging from $N \approx 7$ to $N \approx 10$ for major mergers $(\mu \gtrsim 0.2)$ for final galaxies with $B / T>0.5$, thus showing a weak dependence of $N$ on the descendant stellar mass (the final mass of the growth path) also found in other works (see, e.g., Rodriguez-Gomez et al. 2015 for recent results from the Illustris simulation). Starting from a fractional scatter in $\mathrm{BH}$ masses $\sigma\left(\log M_{\mathrm{BH}, \mathrm{i}}\right) \approx 1$ at very high redshift $z \gtrsim 8$ (see bottom panel of Fig. 4), the effect of merging events (first term in Eq. (4)) is to reduce the fractional scatter to $1 / \sqrt{N} \approx 0.3$, almost independently of the stellar mass. Then we can estimate the contribution to the scatter due to the second term on the right-hand side of Eq. (4), corresponding to the effect of star formation not correlated with the bulge growth (i.e., secular star formation). In fact, the quantities $C \sigma(\epsilon) / \overline{M_{\mathrm{BH}, \mathrm{i}}}$ in Eq. (4) can be recast as $C\left(\sigma(\epsilon) / \dot{M}_{*, \mathrm{MS}}\right)\left(\dot{M}_{*, \mathrm{MS}} / \overline{M_{\mathrm{BH}, \mathrm{i}}}\right)$ where $\dot{M}_{*, \mathrm{MS}}$ is the star formation rate in the main sequence of the $\dot{M}_{*}-M_{*}$ plane (see, e.g., Rodighiero et al. 2011, and references therein; for a comparison of our SAM with the observed main sequence see Lamastra et al. 2013). Since $\sigma(\epsilon)$ is the scatter due to secular star formation, the ratio $\sigma(\epsilon) / \dot{M}_{*, \mathrm{MS}}$ can be approximated by the fractional scatter in the star formation rate of main-sequence galaxies for which typical values $\sigma(\epsilon) / \dot{M}_{*, \mathrm{MS}} \approx 0.3$ are measured (almost independently of the stellar mass, at least up to $z \approx 3$ where measurements are available). Thus, the contribution to the scatter from secular star formation in Eq. (4) can be recast as $3(C / N)\left(\dot{M}_{*, \mathrm{MS}} / M_{\odot} \mathrm{yr}^{-1}\right)\left(10^{8} M_{\odot} / \overline{M_{\mathrm{BH}, \mathrm{i}}}\right) \Delta t / \mathrm{Gyr}$. The relevant quantities can be estimated from the growth paths in the bottom panel of Fig. 3: for initial BH masses $\overline{M_{\mathrm{BH}, \mathrm{i}}} \approx 5 \times 10^{6} M_{\odot}$ and initial stellar masses $M_{*} \lesssim 10^{8} M_{\odot}$, the high-redshift mainsequence star formation rate is $\dot{M}_{*, \mathrm{MS}} \approx 0.1 M_{\odot} \mathrm{yr}^{-1}$ (as results from our SAM, and also from extrapolating to low masses the main-sequence relation at $z \geq 2.5$ in Rodighiero et al. 2011). Adopting $C \approx 1 / 5$ (see the Appendix) and $N \approx 10$ yields a contribution to the scatter from non-bursty star formation $\lesssim 0.06 \Delta t / \mathrm{Gyr}$. Recalling that $\Delta t$ is the growth time of the $\mathrm{BH}$ and stellar mass, the fast growth phase of BHs and bulge masses at $z \gtrsim 2.5$ corresponds to $\Delta t \lesssim 2-3 \mathrm{Gyr}$ (in the paths in the bottom panel of Fig. 3 all events with $\lambda \gtrsim 10^{-3}$ dominating the accretion are concentrated in this epoch). Thus, the final contribution 
to the scatter from non-bursty star formation can be estimated as $\approx 0.1-0.2$. We note that this contribution is - to the first order - weakly dependent on the stellar mass, since higher values of $\dot{M}_{*, \mathrm{MS}}$ corresponding to larger stellar masses are compensated by larger progenitor $\mathrm{BH}$ masses $\overline{M_{\mathrm{BH}, \mathrm{i}}}$, while the number of mergers $N$ is approximately independent of the mass scale, as discussed above.

In summary, for the build-up of classical bulges, we expect contributions from mergers and secular star formation summing up to $\approx 0.4$ and weakly dependent on the stellar mass, as indeed shown in the top histogram in Fig. 3. The evolution of the scatter is initially driven by merging events which reduce it from 1 dex to 0.3 between $z \approx 8$ and $z \approx 3$; in the same period the accretion term in Eq. (4) adds up to only 0.1 dex. After $z \approx 2.5$ the scatter remains close to 0.4 dex. This evolution is consistent with the evolutionary paths in the bottom panel of Fig. 3.

We can now estimate the growth of the scatter expected for the relation between $M_{\mathrm{BH}}$ and the pseudo-bulge mass $M_{*, \mathrm{~PB}}$ shown in Fig. 4, adopting the same line of arguments presented above. In this case, the lower number of major merging events (averaging over the paths in Fig. 4 yields values $N \approx 5$ ), and the longer time-scales involved in $\mathrm{BH}$ formation continuing down to $z=1$ (corresponding to $\Delta t \approx 6 \mathrm{Gyr}$ ) combine to provide a contribution to the fractional scatter $\approx 0.6-0.7$, close to the value obtained from the full semi-analytic model and shown by the histogram at the top of Fig. 4. The same argument can explain the even larger scatter obtained for the relation between $M_{\mathrm{BH}}$ and the disc magnitude shown in Eq. (5). The large values resulting from the model (see the top histogram in Fig. 5) result from the longer time lapse $\Delta t$ now extending from $z \approx 8$ to $z \approx 0$, as shown by the histories in the bottom panel of Fig. 5 .

\section{Conclusions}

Motivated by recent observations indicating that SMBHs correlate differently with the different stellar components of the host galaxies (see Kormendy \& Ho 2013), we use a semi-analytic model of galaxy formation to investigate the correlation of the different galaxy stellar components with the mass of the central SMBH (for a parallel study of the role of galaxy morphology in determining the spin distribution of SMBHs, see Sesana et al. 2014). The stellar mass in the disc, bulge, and pseudo-bulge of galaxies is related to quiescent star formation, galaxy interactions, and the loss of angular momentum following disc instabilities, respectively.

Although the model we adopt includes both interactions and disc instabilities in isolated galaxies as possible triggers for $\mathrm{BH}$ accretion, even assuming the highest possible contribution from disc instabilities the bulk of the final $\mathrm{BH}$ mass $M_{\mathrm{BH}}$ is thus contributed by interactions. These are also responsible for the growth of the mass $M_{*, B}$ of classical bulges and - in CDM models - are expected to take place at large redshift. Such a parallel evolution of $M_{\mathrm{BH}}$ and of $M_{*, B}$ within a short time lapse $(z \gtrsim 3)$ yields the tight correlation between BHs and the bulge stellar mass. Our assumed modelling for disc instabilities naturally yields an appreciably larger scatter for the relation between $M_{\mathrm{BH}}$ and the masses of pseudo-bulges $M_{*, \mathrm{~PB}}$. In fact, in our model the latter are entirely contributed by disc instabilities (a widely adopted view, implemented in other SAMs; see e.g., Fontanot et al. 2015a), which are favoured in gas-rich galaxies. The latter feature implies that the growth of a substantial pseudo-bulge must take place along those histories characterized by a low number of major merging at high redshifts in order to avoid the consumption of gas occurring in major mergers at early times. The longer time scales involved in the growth of pseudobulges, along with the decoupled growth of $M_{*, \mathrm{~PB}}$ and $M_{\mathrm{BH}}$ (still mainly contributed by galaxy interactions) lead to the large scatter in the predicted $M_{\mathrm{BH}}-M_{*, \mathrm{~PB}}$ relation. The longer time scales and decoupled growth with respect to the BHs also characterize the growth of the disc stellar component $M_{*, D}$ (star formation time-scales $\sim 1 \mathrm{Gyr}$ ), causing a large scatter and a weak correlation (or none at all) between $M_{\mathrm{BH}}$ and $M_{*, D}$. The extreme instance of such a decoupled and delayed star formation with respect to the $\mathrm{BH}$ growth is constituted by bulgeless galaxies.

Our model does not yield a steepening of the slope of the $a v$ erage $M_{\mathrm{BH}}-M_{*, B}$ at low $\mathrm{BH}$ masses reported by some authors (see Graham \& Scott 2015) for $M_{\mathrm{BH}} \lesssim 2 \times 10^{8} M_{\odot}$, who proposed that such a break in the power-law scaling of $M_{\mathrm{BH}}$ with the bulge mass may indicate AGN feedback as the main driver for the observed relations. While on the observational side the presence of such a break in the power-law behaviour is still debated (as opposed to a larger scatter, see Kormendy \& Ho 2013), on the theoretical side our results agree with the findings of other SAM (Fontanot et al. 2015b) in predicting that disc instabilities, AGN feedback, or other processes considered in the present paper do not provide a steepening in the slope of the $M_{\mathrm{BH}}-M_{\mathrm{sph}}$ relation when the peak BH mass (for any given $M_{\mathrm{sph}}$ ) is considered. The model in Fontanot et al. (2015b) yields a steepening for $M_{\mathrm{BH}} \lesssim 10^{8} M_{\odot}$ only when it includes stellar feedback in star-forming bulges (a process not included in our model). On the other hand, we predict an increase in the scatter for the correlation between $M_{\mathrm{BH}}$ and the total stellar mass $M_{*}$ for low-mass galaxies (see, e.g., Menci et al. 2014) due to the larger contribution of the pseudo-bulge and disc components to the total stellar content in such objects; the skewness toward small BH masses of such a scatter due to the contribution of pseudo-bulges may explain the steepening in the $M_{\mathrm{BH}}-M_{\text {sph }}$ relation reported by Graham \& Scott (2015).

Although in some phenomenological models a $M_{\mathrm{BH}}-M_{*, B}$ relation characterized by a single power law can result from dry mergers driving $\mathrm{BHs}$ and their hosted galaxies towards a mean relation (Peng 2007; Hirschmann et al. 2010; Jahnke \& Macció 2011), in our model the $\mathrm{BH}$ growth is mainly contributed by $\mathrm{BH}$ accretion, and the $M_{\mathrm{BH}}-M_{*}$ relations results from the correlated $\mathrm{BH}$ and bulge growth from a common gas supply. The final $M_{\mathrm{BH}}-M_{*, B}$ relation stems from the physical relation between such an accretion and the build-up of the bulge component in a short time lapse, followed by a phase $(z \lesssim 3)$ in which the gas content of galaxies, their merging rate, and the $\mathrm{BH}$ accretion drop. A consistency check for such a framework is indeed constituted by the $\mathrm{BH}$ relation with the stellar mass which forms in the subsequent phase $(z \lesssim 3)$, i.e., the disc and pseudo-bulge components: their weaker correlation with the $\mathrm{BH}$ mass indicate that the secular processes involved in their formation differ from the impulsive processes driving the build-up of SMBHs and the bulge in the earlier stages of galaxy evolution.

In our model, the establishment of the correlation between the $\mathrm{BH}$ mass and the different stellar components is determined by how the growth of these components is connected to the common gas supply. Changing the efficiency of AGN feedback has a minor effect on the predicted correlations presented in Sect. 3, as also obtained in other SAMs (see, Fontanot et al. 2015b, and discussion therein). Of course, in our model, feedback affects both star formation and $\mathrm{BH}$ accretion, but it has a similar effect on both so that it does not constitute the driver of the $M_{\mathrm{BH}}-M_{*}$ relations. Although the feedback origin of the connection between 
black holes and galaxies has been proposed or postulated in several analytical models (see, e.g., Silk \& Rees 1998; Fabian et al. 1999; King 2003, 2005; Whythe \& Loeb 2003), we note that to establish the origin of the BH-galaxy correlation a detailed description of the relative growth of the $\mathrm{BH}$ and stellar components is needed. This, in turn, requires a detailed treatment of the several non-linear processes (gas cooling, disc formation, galaxy interactions) to connect them with the cosmological growth of the host galaxies, a task best accomplished through SAMs or $\mathrm{N}$-body simulations. While in some simulations (see Di Matteo et al. 2005, 2008; Debuhr 2011; Choi et al. 2012; see also Costa et al. 2014) AGN feedback is required to establish the $M_{\mathrm{BH}}-M_{*}$ relation, all such simulations assume Bondi accretion for the $\mathrm{BH}$ growth. Indeed, as already noted by Angles-Alcazar et al. (2016), in any model where the dependence of the BH accretion rate on $M_{\mathrm{BH}}$ is more than linear (as in the Bondi accretion, scaling like $M_{\mathrm{BH}}^{2}$ ) a divergent evolution of the $\mathrm{BH}$ mass would be obtained in the absence of a strong feedback. Thus, in such simulations, the need for feedback stems from the assumed accretion rate. In simulations where a milder dependence on $M_{\mathrm{BH}}$ is assumed for BH accretion (see Angles-Alcazar et al. 2016), the establishment of the $M_{\mathrm{BH}}-M_{*}$ relations is not initiated by the feedback but rather results from the relative growth of the stellar and BH components.

Our model provides, in principle, testable expectations for the evolution of the relation of $\mathrm{BH}$ masses with the different stellar components. Inspection of the paths $M_{\mathrm{BH}}(t)-M_{*, B}(t)$ in the bottom panel of Fig. 3 shows that for galaxies dominated by bulges the typical paths lie above the local relation, so we expect for such galaxies (and in general for massive galaxies formed from the collapse of biased regions of the density field) a steeper $M_{\mathrm{BH}}-M_{*, B}$ relation at high redshifts (see Lamastra et al. 2010) consistent with available observations of quasars extending up to $z \approx 4$ (Walter et al. 2004; Riechers et al. 2008). On the other hand, the evolutionary paths of $M_{\mathrm{BH}}(t)-M_{*, B}(t)$ for the pseudo-bulges and disc components (bottom panels of Figs. 4 and 5) show a mild trend toward lying below the local relation. Although at present observational limitations do not allow the evolution of the relation of $\mathrm{BH}$ masses with the different stellar components to be explored, future measurements of such evolutionary paths for different stellar components will provide a stringent probe for the role of the different $\mathrm{BH}$ feeding processes in determining the growth of BHs.

Finally, we discuss the effects of relaxing the assumptions of our fiducial modelling on the relation between the $\mathrm{BH}$ mass and the different stellar components. The results presented above assume that classical bulges are formed from major mergers (see Sect. 2.2). However, some authors argue that the local frequency of spirals with low values of the $B / T$ ratio $(B / T \lesssim 0.2)$ may indicate that secular processes can appreciably affect the formation of classical bulges (see, e.g., Weinzirl et al. 2009; Saha 2015), a possibility explored in some theoretical works based on SAMs (see Perry et al. 2009; De Lucia et al. 2011; see also Brenman et al. 2015). The effect of assuming such a hypothesis can be explored in our model by assuming that all bulges are formed through the disc instability process (case iii in Sect. 2.2). The result of such an assumption would be a relation between BH mass and classical bulge mass $M_{\mathrm{BH}}-M_{*, B}$ similar to that shown in Fig. 4, characterized by a large dispersion and very weak correlation. Although present data seem to exclude such a case (see, e.g., Kormendy \& Ho 2013), if future observational improvements (or a refined treatment of observational biases, see Shankar et al. 2016) should lead to a revision of the present observational picture this would constitute strong support for the disc instability origin of the majority of galaxy bulges. Another possible variant of our model is constituted by assuming that disc instabilities constitute the main contribution to the $\mathrm{BH}$ accretion. Within our physical modelling of disc instabilities (Sect. 2), such a feeding mode can indeed be comparable to galaxy interactions for intermediate AGN luminosity (bolometric luminosity $10^{44} \lesssim L_{\mathrm{Bol}} / \mathrm{erg} \mathrm{s}^{-1} \lesssim 10^{46}$ ) at $z \approx 1.5-2.5$ (see Menci et al. 2014; Gatti et al. 2015). In the extreme case of a dominance of disc instability as $\mathrm{BH}$ accretion triggers in the above redshift range, we expect a decrease in the dispersion in the correlation $M_{\mathrm{BH}}-M_{*, \mathrm{~PB}}$ between the $\mathrm{BH}$ mass and the pseudo-bulge mass at $z \approx 1-2$ since the growth of both quantities would be driven by the same physical process: at the same time, we would expect a larger dispersion in the relation of $\mathrm{BH}$ masses with the masses of classical bulges. In the future, measurements of such a correlation in the above redshift interval will contribute probing the relative role of interactions and disc instabilities in triggering the $\mathrm{BH}$ accretion, in the regimes where disc instability may provide their maximum contribution.

Finally, we note that additional mechanisms not implemented in our SAM may play a role in $\mathrm{BH}$ feeding at high redshifts, such as violent disc instabilities (Bournaud et al. 2011) or even direct formation of a compact bulge from the cosmic web (Dubois et al. 2012). We plan to investigate the effects of such mechanisms in future works.

Acknowledgements. This work was partially supported by PRIN INAF 2011. We thank the referee for constructive comments that helped to improve the manuscript.

\section{References}

Anglés-Alcázar, D., Özel. F., \& Davé, R. 2013, ApJ, 770, 5

Anglés-Alcázar, D., Davé, R., Faucher-Giguere, C.-A., et al. 2016, MNRAS, submitted [arXiv: 1603.08007]

Athanassoula, E. 2005, MNRAS, 358, 1477

Avila-Reese, V., Zavala, J., \& Lacerna, I. 2014, MNRAS, 441, 417

Bardeen, J. M., Bond, J. R., Kaiser, N., \& Szalay, A. S. 1986, ApJ, 304, 15

Beifiori, A., Courteau, S., Corsini, E. M., \& Zhu, Y. O. 2012, MNRAS, 419, 2497

Bennert, V. N., Auger, M. W., Treu, T., Woo, J.-H., \& Malkan, M. A. 2011, ApJ, 742, 107

Bournaud, F., Dekel, A., Teyssier, R., et al. 2011, ApJ, 741, L33

Bower, R. 1991, MNRAS, 248, 332

Brenman, R., Pandya, V., Somerville, R. S., et al. 2015, MNRAS, 451, 2933

Bruzual, G., \& Charlot, S. 2003, MNRAS, 344, 1000

Cappellari, M., Bacon, R., Bureau, M., et al. 2006, MNRAS, 366, 1126

Cattaneo, A., Blaziot, J., Devriendt, J., \& Guiderdoni, B. 2005, MNRAS, 364, 407

Croton, D. J., Springel, V., White, S. D. M., et al. 2006, MNRAS, 365, 11 Choi, E., Ostriker, J. P., Naab, T., \& Johansson, P. H. 2012, ApJ, 754, 125 Cisternas, M., Jahnke, K., Bongiorno, A., et al. 2011, ApJ, 741, 11

Combes, F., Debbasch, F., Friedli, D., \& Pfenniger, D., 1990, A\&A, 233, 82 Costa, T., Sijacki, D., \& Haenhelt, M. G. 2014, MNRAS, 444, 2355

Debuhr, J., Quataert, E., \& Ma, C.-P. 2011, MNRAS, 412, 1341

Debattista, V. P., Mayer, L., Carollo, C. M., et al. 2006, ApJ, 645, 209

De Lucia, G., Fontanot, F., Wilman, D., \& Monaco, P. 2011, MNRAS, 414, 1439

Di Matteo, T., Springel, V., \& Hernquist, L. 2005, Nature, 433, 604

Di Matteo, T., Colberg, J., Springel, V., Hernquist, L., \& Suacki, D. 2008, ApJ, 676,33

Dubois, Y., Pichon, C., Haehnelt, M., et al. 2012, MNRAS, 423, 361

Efstathiou, G., Lake, G., \& Negroponte, J. 1982, MNRAS, 199, 1069

Eliche-Moral, M. C., Balcells, M., Aguerri, J. A. L., \& Gonzalez-Garcia, A. C. 2006, A\&A, 457, 91

Fabian, A. C. 1999, MNRAS, 308, L39

Fanidakis, N., Baugh, C. M., Benson, A. J., et al. 2012, MNRAS, 419, 2797

Ferrarese, L., \& Merritt, D. 2000, ApJ, 539, L9

Fontanot, F., Monaco, P., Cristiani, S., \& Tozzi, P. 2006, MNRAS, 373, 1173

Fontanot, F., Macció, A. V., Hirschmann, G., et al. 2015a, MNRAS, 451, 2968

Fontanot, F., Monaco, P., \& Shankar, F. 2015b, MNRAS, 453, 4112

Gadotti, D. A. 2009, MNRAS, 393, 1531 
Gatti, M., Lamastra, A., Menci, N., \& Fiore, F. 2015, A\&A, 576, A32

Gebhardt, K., Bender, R., Bower, G., et al. 2000, ApJ, 539, L13

Graham, A. W. 2007, MNRAS, 379, 711

Graham, A. W., \& Li, I.-H. 2009, ApJ, 698, 812

Graham, A. W., \& Scott, N. 2013, ApJ, 764, 151

Graham, A. W., \& Scott, N. 2015, ApJ, 798, 54

Graham, A. W., Onken, C. A., Athanassoula, E., \& Combes, F. 2011, MNRAS 412,2211

Greene, J. E., \& Ho, L. C. 2006, ApJ, 641, 121

Greene, J. E., Peng, C. Y., Kim, M., et al. 2010, ApJ, 721, 26

Granato, G. L., De Zotti, G., Silva, L., Bressan, A., \& Danese, L. 2004, ApJ, 600,580

Gultekin, K., Richstone, D. O., Gebhardt, K., et al. 2009, ApJ, 698, 198

Hirschmann, M., Khochfar, S., Burkert, A., et al. 2010, MNRAS, 407, 1016

Hirschmann, M., Somerville, R. S., Naab, T., \& Burkert, A. 2012, MNRAS, 426, 237

Hirschmann, M., Dloag, K., Saro, A., et al. 2014, MNRAS, 442, 2304

Hu, J. 2008, MNRAS, 386, 2242

Häring, N., \& Rix, H.-W. 2004, ApJ, 604, L89

Henriques, B. M. B., \& Thomas, P. A. 2010, MNRAS, 403, 768

Hopkins, P. F., \& Quataert, E. 2011, MNRAS, 411, 1027

Hopkins, P. F. Hernquist, L., Cox, T. J., et al. 2006, ApJS, 163, 1

Hopkins, P. F., Somerville, R. S., Cox, T. J., et al. 2009, MNRAS, 397, 802

Jahnke, K., \& Macció, A. V., 2011, ApJ, 734, 92

Kauffmann, G., \& Haehnelt, M., 2000, MNRAS, 311, 576

Keselman, J. A., \& Nusser, A. 2012, MNRAS, 424, 1232

King, A. 2003, ApJ, 596, L27

Kormendy, J. 2013, in XXIII Canary Islands Winter School of Astrophysics, Secular Evolution of Galaxies, eds. J. Falcon-Barroso, \& J. H. Knapen (Cambridge: Cambridge Univ. Press), 1

Kormendy, J., \& Ho, J. C. 2013, ARA\&A, 51, 511

Kormendy, J., \& Kennicutt, R. C. 2004, ARA\&A, 42, 603

Kormendy, J., \& Richstone, D. 1995, ARA\&A, 33, 581

Kormendy, J., Bender, R., \& Cornell, M. E. 2011, Nature, 469, 374

Kuo, C. Y., Braatz, J. A., Condon, J. J., et al. 2011, ApJ, 727, 20

Lacey, C., \& Cole, S. 1993, MNRAS, 262, 627

Lamastra, A., Menci, N., Maiolino, R., Fiore, F., \& Merloni, A. 2010, MNRAS, 405, 29

Lamastra, A., Menci, N., Fiore, F., \& Santini, P. 2013, MNRAS, 552, 44

Läsker, R., Greene, J. E., Seth, A., et al. 2016, ApJ, 825, 3

Lo Faro, B., Monaco, P., Vanzella, E., et al. 2009, MNRAS, 399, 827

Magorrian, J., Tremaine, S., Richstone, D., et al. 1998, AJ, 115, 2285

Marconi, A., \& Hunt, L. K., 2003, ApJ, 589, L21

McConnell, N. J., \& Ma, C.-P. 2013, ApJ, 764, 184

McConnell, N. J., Ma, C.-P., Gebhardt, K., et al. 2011, Nature, 480, 215

Menci, N., Cavaliere, A., Fontana, A., et al. 2003, ApJ, 587, L63

Menci, N., Fiore, F., Puccetti, S., \& Cavaliere, A. 2008, ApJ, 686, 219
Menci, N., Gatti, M., Fiore, F., \& Lamastra, A. 2014, A\&A, 569, 37

Mendez-Abreu, J., Debattista, V. P., Corsini, E. M., \& Aguerri, J. A. L. 2014 A\&A, 572, A25

Merritt, D., \& Ferrarese, L. 2001, ApJ, 547, 140

Mo, H. J, Mao, S., \& White, S. D. M. 1998, MNRAS, 295, 319

Monaco, P., Salucci, P., \& Danese, L. 2000, MNRAS, 311, 279

Obreja, A., Domínguez-Tenreiro, R., Brook, C., et al. 2013, ApJ, 763, 26

Parry, O. H., Eke, V. R., \& Frenk, C. S. 2009, MNRAS, 396, 1972

Peng, C. Y. 2007, ApJ, 671, 1098

Peterson, B. M., Bentz, M. C., Desroches, L.-B., et al. 2005, ApJ, 632, 799; Erratum. 2005, ApJ, 641, 638

Raha, N., Sellwood, J. A., James, R. A., \& Kahn, F. D. 1991, Nature, 352, 411

Reines, A. E., Sivakoff, G. R., Johnson, K. E., \& Brogan, C. L. 2011, Nature, 470, 66

Riechers, D. A., Walter, F., Carilli, C. L., Bertoldi, F., \& Momjian, E., 2008, ApJ, 686, L9

Rodighiero, G., Daddi, E., Baronchelli, I., et al. 2011, ApJ, 739, L40

Rodriguez-Gomez, V., Genel, S., Vogelsberger, M., et al. 2015, MNRAS, 449, 49

Saha, K. 2015, ApJ, 806, L29

Sanders, D. B., \& Mirabel, I. F. 1996, ARA\&A, 34, 749

Sani, E., Marconi, A., Hunt, L. K., \& Risaliti, G. 2011, MNRAS, 413, 1479

Santini, P., Maiolino, R., Magnelli, B., et al. 2014, A\&A, 562, A30

Schweizer, F. 1990, in Dynamics and Interactions of Galaxies, ed. R. Wielen (New York: Springer), 60

Sesana, A., Barausse, E., Dotti, M., Rossi, M. 2014, ApJ, 794, 104

Shankar, F., Marulli, F., Mathur, S., Bernardi, M., \& Bournaud, F. 2012, A\&A, 540, A23

Shankar, F., Weinberg, D. H., \& Miralda-Escude, J. 2013, MNRAS, 428, 421

Sijacki, D., Vogelsberger, M., Genel, S., et al. 2015, MNRAS, 452, 575

Silk, J., \& Mamon, G. A. 2012, ARA\&A,12, 917

Silk, J., \& Rees, M. J. 1998, A\&A, 331, L1

Shankar, F., Bernardi, M., Sheth, R. K., et al. 2016, MNRAS, 460, 3119

Somerville, R. S., \& Davé 2015, ARA\&A, 53, 41

Thornton, C. E., Barth, A. J., Ho, L. C., Rutledge, R. E., \& Greene, J. E. 2008, ApJ, 686, 892

Toomre, A. 1977, in The Evolution of Galaxies and Stellar Populations, eds. B. M. Tinsley, \& R. B. Larson (New Haven: Yale University Observatory), 401 Toomre, A., \& Toomre, J. 1972, ApJ, 178, 623

Tremaine, S., Gebhardt, K., Bender, R., et al. 2002, ApJ, 574, 740

Volonteri, M., Dubois, Y., Pichon, C., \& Devriendt, J. 2016, MNRAS, 460, 2979

Walter, F., Carilli, C., Bertoldi, F., et al. 2004, ApJ, 615, L17

Weinzirl, T., Jogee, S., Khochfar, S., Burkert, A., Kormendy, J. 2009, ApJ, 696, 411

Wyse, R. F G., Gilmore, G., \& Franx, M. 1997, ARA\&A, 35, 637

Zhao, D. H., Mo, H. J., Jing, Y. P., \& Börner, G. 2003, MNRAS, 339, 12 


\section{Appendix A}

We consider the toy model introduced in Sect. 4, which is meant to provide a simplified analytical rendition of the growth of the stellar and BH masses of our SAM. In the toy model, galaxies are characterized by identical initial stellar masses $M_{*, i}$ each hosting a $\mathrm{BH}$ mass $M_{\mathrm{BH}, \mathrm{i}}$ uncorrelated with their host mass. A given, fixed stellar mass growth $\Delta M_{*}$ in a time interval $\Delta t$ is contributed by:

$\Delta M_{*}=\Sigma_{i}^{N} M_{*, i}+\Delta M_{*, \text { bursts }}+\epsilon \Delta t$.

The first term corresponds to the merging of $N$ galaxies with initial stellar mass $M_{*, i}$; the second to the growth due to starbursts, the component connected to the BH accretion in our SAM; the third term corresponds to the conversion of gas into stars not correlated to the $\mathrm{BH}$ feeding, with the stochastic variable $\epsilon$ providing a simplified description of the distribution of star formation rate contributed by the secular processes (e.g., the quiescent star formation rate $M_{\mathrm{c}} / \tau_{\mathrm{d}}$ discussed in Sect. 2.1).

While the third term is taken to be uncorrelated to the $\mathrm{BH}$ growth, we assume the starburst term to be correlated to the $\mathrm{BH}$ accreted mass $\Delta M_{\mathrm{BH}}=C \Delta M_{* \text {,bursts }}$; this mimics the description of interaction-driven $\mathrm{BH}$ feeding and starburst of our full SAM, described in Sect. 2. We note that, physically, the constant $C$ relating the $\mathrm{BH}$ accretion rate to the starburst takes values smaller than one (typical values in local ULRIGS values $C \approx 1 / 5-1 / 10$ are observed, Sanders \& Mirabel 1996). The limit $C \rightarrow 0$ corresponds to vanishing $\mathrm{BH}$ accretion.

The accreted $\mathrm{BH}$ mass increment is the difference between the final $\mathrm{BH}$ mass and the sum of the initial mass of the $\mathrm{BH}$ hosted in the progenitor galaxies. Thus $\Delta M_{\mathrm{BH}}=C \Delta M_{* \text {,burst }}=$ $M_{\mathrm{BH}, \mathrm{f}}-\Sigma_{i}^{N} M_{\mathrm{BH}, \mathrm{i}}$. Substituting the latter expression into Eq. (A.1) yields

$M_{\mathrm{BH}, \mathrm{f}}=\Sigma_{i}^{N} M_{\mathrm{BH}, \mathrm{i}}+C \Delta M_{*}-C \Sigma_{i}^{N} M_{*, i}-C \epsilon \Delta t$.

The average final BH mass corresponding to Eq. (A.2) is then

$\overline{M_{\mathrm{BH}, \mathrm{f}}}=N \overline{M_{\mathrm{BH}, \mathrm{i}}}+C \Delta M_{*}-C N \overline{M_{*, i}}-C \bar{\epsilon} \Delta t$,

while the dispersion (square root of the variance) is

$\sigma\left(M_{\mathrm{BH}, \mathrm{f}}\right)=\sqrt{N} \sigma\left(M_{\mathrm{BH}, \mathrm{i}}\right)+C \sigma(\epsilon) \Delta t$,

where $\sigma(\epsilon)$ is the variance generated by all star formation processes not correlated to the BH accretion. From Eqs. (A.3) and (A.4) we obtain the fractional dispersion as

$\sigma\left(\log M_{\mathrm{BH}, \mathrm{f}}\right)=\frac{\sigma\left(M_{\mathrm{BH}, \mathrm{f}}\right)}{\overline{M_{\mathrm{BH}, \mathrm{f}}}}=\frac{\sigma\left(M_{\mathrm{BH}, \mathrm{i}}\right)+(1 / \sqrt{N}) C \sigma(\epsilon) \Delta t}{\sqrt{N} \overline{M_{\mathrm{BH}, \mathrm{i}}}+(1 / \sqrt{N}) \overline{\Delta M_{\mathrm{BH}}}}$

Taylor expansion of Eq. (A.5) for values $(1 / \sqrt{N}) \rightarrow 0$ yields

$\sigma\left(\log M_{\mathrm{BH}}, f\right)=\frac{\sigma\left(\log M_{\mathrm{BH}, \mathrm{i}}\right)}{\sqrt{N}}+\frac{C \sigma(\epsilon) \Delta t}{N \overline{M_{\mathrm{BH}, \mathrm{i}}}}$.

The first term corresponds to the statistical suppression of the fractional dispersion pointed out by Peng (2007), which is recovered in the limit of vanishing $\mathrm{BH}$ accretion $C \rightarrow 0$. The second term in Eq. (A.6) is generated by the star formation uncorrelated to the $\mathrm{BH}$ growth. 